ARTICLE

https://doi.org/10.1038/s41467-018-07790-x

\title{
Overall water splitting by graphdiyne-exfoliated and -sandwiched layered double-hydroxide nanosheet arrays
}

\author{
Lan Hui ${ }^{1,2}$, Yurui Xue (1) ${ }^{1,3}$, Bolong Huang (10 ${ }^{4}$, Huidi Yu', Chao Zhang ${ }^{1}$ \\ Danyan Zhang ${ }^{1}$, Dianzeng Jia ${ }^{2}$, Yingjie Zhao ${ }^{1,3}$, Yongjun Li (i) ${ }^{1}$, Huibiao Liu ${ }^{1}$ \& Yuliang Li (i) 1,3,5
}

It is of great urgency to develop efficient, cost-effective, stable and industrially applicable electrocatalysts for renewable energy systems. But there are still few candidate materials. Here we show a bifunctional electrocatalyst, comprising graphdiyne-exfoliated and -sandwiched iron/cobalt layered double-hydroxide nanosheet arrays grown on nickel foam, for the oxygen and hydrogen evolution reactions. Theoretical and experimental data revealed that the charge transport kinetics of the structure were superior to iron/cobalt layered doublehydroxide, a prerequisite for improved electrocatalytic performance. The incorporation with graphdiyne increased the number of catalytically active sites and prevented corrosion, leading to greatly enhanced electrocatalytic activity and stability for oxygen evolution reaction, hydrogen evolution reaction, as well as overall water splitting. Our results suggest that the use of graphdiyne might open up new pathways for the design and fabrication of earthabundant, efficient, functional, and smart electrode materials with practical applications.

\footnotetext{
${ }^{1}$ Key Laboratory of Organic Solids, Institute of Chemistry, Chinese Academy of Sciences, Beijing 100190, P. R. China. ${ }^{2}$ Key Laboratory of Energy Materials Chemistry, Ministry of Education; Key laboratory of Advanced Functional Materials, Autonomous Region; Institute of Applied Chemistry, Xinjiang University, Urumqi 830046, P. R. China. ${ }^{3}$ School of Polymer Science and Engineering, Qingdao University of Science and Technology, Qingdao 266042, P. R. China. ${ }^{4}$ Department of Applied Biology and Chemical Technology, The Hong Kong Polytechnic University, Hong Kong, P. R. China. ${ }^{5}$ University of Chinese Academy of Sciences, Beijing 100049, P. R. China. Correspondence and requests for materials should be addressed to Y.X. (email: xueyurui@iccas.ac.cn) or to D.J. (email: jdz@xju.edu.cn) or to Y.L. (email: ylli@iccas.ac.cn)
} 
T he electrochemical or photoelectrochemical splitting of water into hydrogen and oxygen is a sustainable technology for energy conversion and storage ${ }^{1-4}$. Among the drawbacks of applying these approaches industrially are the sluggish kinetics of the oxygen and hydrogen evolution reactions (OER/HER). Thus, there remains great demand for efficient, costeffective, stable, and industrially applicable electrocatalysts for overall water splitting (OWS). At present, precious metals provide the state-of-the-art electrocatalysts for water splitting (e.g., $\mathrm{IrO}_{2}$ and $\mathrm{RuO}_{2}$ for OER; Pt for HER), but their use on large scales is hampered by their high cost and scarcity. Extensive efforts are, therefore, being devoted to the development of earth-abundant electrocatalysts, including first-row (3d) transitional metal (TM) layered double hydroxides $(\mathrm{LDH})^{5,6}$, oxides, nitrides ${ }^{7}$, and sulfides $^{8}$. Owing to their structural flexibility and chemical versatility, TM LDHs have emerged as promising alternatives to their precious metal counterparts, and multi-metal LDHs are particularly interesting because their intrinsic activities generally outperform those of their mono-metal counterparts. Nevertheless, the low conductivity and poor electroactivity and stability of bulk LDHs (b-LDHs) have severely impeded their potential applications $s^{5}$. Attempts have been made to solve these problems by exfoliating thick b-LDHs into ultrathin nanosheets, directly growing LDHs onto conductive substrates, or hybridizing LDHs with conductive carbon materials ${ }^{5,9-12}$. On the other hand, coating catalysts with two-dimensional (2D) carbon materials can also be an effective means of designing new electrocatalysts with improved activities and stabilities ${ }^{12}$. These approaches have been and are being used in the synthesis of LDH-based electrocatalysts, but with significant limitations. For example, although exfoliated LDHs (e-LDHs) possess more active sites and higher electronic conductivities than pristine $\mathrm{LDHs}^{9}$, they must be bonded onto supports using polymer binders (e.g., Nafion/PTFE) when fabricating electrode materials, thereby resulting in significant degradation of the conductivity, activity, and structural stability of the electrode-the result of decreased ion/electron transport kinetics and decomposition of the catalysts. Directly growing LDHs onto substrates can obviate the need for binders ${ }^{5,10,11}$, but the previously reported LDHs grown on substrates have always been relatively thick. Accordingly, we suspected that it would be beneficial to combine the above mentioned advantages to develop high-performance electrode materials. We are, however, to the best of our knowledge, unaware of any such previous attempts.

The all-carbon material graphdiyne (GDY) $-\mathrm{a} 2 \mathrm{D}$ one-atomthick sp/sp ${ }^{2}$ co-hybridized carbon network possessing silicon-like electronic conductivity ${ }^{13,14}$, a natural band gap, and natural pores $(2.5 \AA)^{15}$ - appears to be a material that has the ability to resolve several of the problems affecting current electronic and energy materials when applied in various fields (e.g., electrocatalysis, energy storage, optical devices, electronic devices), the result of its unique structural, physical, and chemical properties ${ }^{16-27}$. More importantly, GDY is the only carbon materials that can be synthesized in a controllable and facile manner on arbitrary substrates. In a typical reaction process, hexaethynylbenzene (HEB) monomers first interact with the substrate and self-assemble into a monolayer on its surface, followed by in situ polymerization to form GDY ${ }^{16}$. For LDHs, the large interlayer distance and high exchangeability of the interlayer anions make it easy for HEB monomers to enter into the LDH gallery. Coupling of the alkynyl units would then occur within the confined spaces, leading to the simultaneous formation of GDY layers on both sides of the LDH surfaces. Owing to the flexibility of both GDY and LDH, the stress/deformation caused by their intimate contact would further enlarge the layer spacing, leading to complete exfoliation of the b-LDHs into thicker LDH nanosheets.

This creative strategy has many significant advantages over more traditional ways of designing and fabricating efficient electrocatalysts. First, the intrinsically high electrical conductivity of GDY can effectively facilitate charge transport and improve electrocatalytic kinetics and performances. Another attractive property of GDY is that its high content of triple bonds makes it rich in charged carbon atoms, which can provide more active sites and, thus, better catalytic activity. Furthermore, GDY appears to be one of the most stable diacetylenic carbon materials; thus, it can effectively protect electrocatalysts from corrosion and greatly improve their long-term stability 28,29 . We suspected that our proposed in situ growth strategy would provide direct and seamless contacts between GDY and LDH, without the need for any polymer binders, thereby decreasing the resistance, improving the charge transfer behavior, and facilitating catalysis. Furthermore, the naturally porous structure of GDY and the large open spaces in the catalyst would be beneficial to efficient mass transport, gas evolution, and the efficient use of active sites, thereby greatly improving the system's catalytic performance.

Herein, we describe the in situ exfoliation and modification of bulk iron-cobalt LDH nanosheets through a GDY-induced intercalation/exfoliation/decoration strategy (e-ICLDH@GDY/ $\mathrm{NF}$ ). This approach allows us not only to exfoliate thick bulkLDHs into ultrathin e-LDHs in situ but also simultaneously form GDY sandwiched structures. Compared with catalytically inert bulk-LDHs, the e-ICLDH@GDY/NF structure displays greatly enhanced electrocatalytic activities and stabilities for the OER and HER in $1.0 \mathrm{M} \mathrm{KOH}$. Furthermore, this structure requires only low cell voltages of $1.43,1.46$, and $1.49 \mathrm{~V}$ to provide current densities of 10,100 , and $1000 \mathrm{~mA} \mathrm{~cm}^{-2}$, respectively, for OWS.

\section{Results}

Synthesis and structural characterization. Figure 1 provides a schematic representation of the synthesis of the electrocatalyst (please see Methods section for details). The self-supported iron-cobalt LDH (ICLDH) nanosheet array was first grown on nickel foam (NF) simply through hydrothermal treatment, followed by a cross-coupling reaction ${ }^{30,31}$. In general, the anions located in the interlayer regions can be easily replaced. And the large interlayer distance in LDH make it easy for the entrance of HEB monomers into the LDHs gallery. The acetylenic hydrogen of HEB could then form hydrogen bonds with the hydroxide layer, which can lead to the formation of HEB film on the LDH surfaces. A Glaser-Hay reaction would then take place efficiently with the aid of TMEDA. The GDY films could be uniformly grown on LDH surfaces. Owing to the flexibility nature of both GDY and LDH, the stress/deformation caused by the intimate contact in-between will further enlarge the layer spacing, leading to the complete exfoliation of b-LDHs into thicker $\mathrm{LDH}$ nanosheets $(\mathrm{e}-\mathrm{LDH})$. The resulted product is denoted here as e-LDH@GDY/NF.

We used scanning electron microscopy (SEM) to study the morphologies of the samples. For pure CLDH, a nanowire morphology, several micrometers long, formed on the NF surface (Fig. 2a, b); pure ILDH also had a nanosheet morphology, but with an average size of approximately $0.5 \mu \mathrm{m}$ (Fig. 2c, d). After the incorporation of $\mathrm{Co}$ and $\mathrm{Fe}$ atoms, the ICLDH structure exhibited a nanosheet morphology similar to that of ILDH; it was grown vertically and uniformly on the surface of the NF (Fig. 2e-h). These ICLDH nanosheets possessed a smooth surface, a thickness of approximately $40-80 \mathrm{~nm}$, and a larger lateral size of approximately $1.5 \mu \mathrm{m}$ (Fig. $2 \mathrm{~h}$ ). After crosscoupling, the samples maintained their nanosheet morphology (Fig. 2i-l) but became much thinner $($ ca. $7.0 \mathrm{~nm})$ and more wrinkled than the pristine ICLDH nanosheets. This result was further verified by atomic force microscopy (AFM) images (Fig. $2 \mathrm{~m}, \mathrm{o}$ ) and corresponding height profiles (Fig. $2 \mathrm{n}, \mathrm{p}$ ). In order to confirm whether the morphologies of the LDHs in pyridine or TMEDA can be damaged, pristine LDHs were put into the reactor containing pyridine or TMEDA only (without HEB). Other experimental conditions were the same as that used 


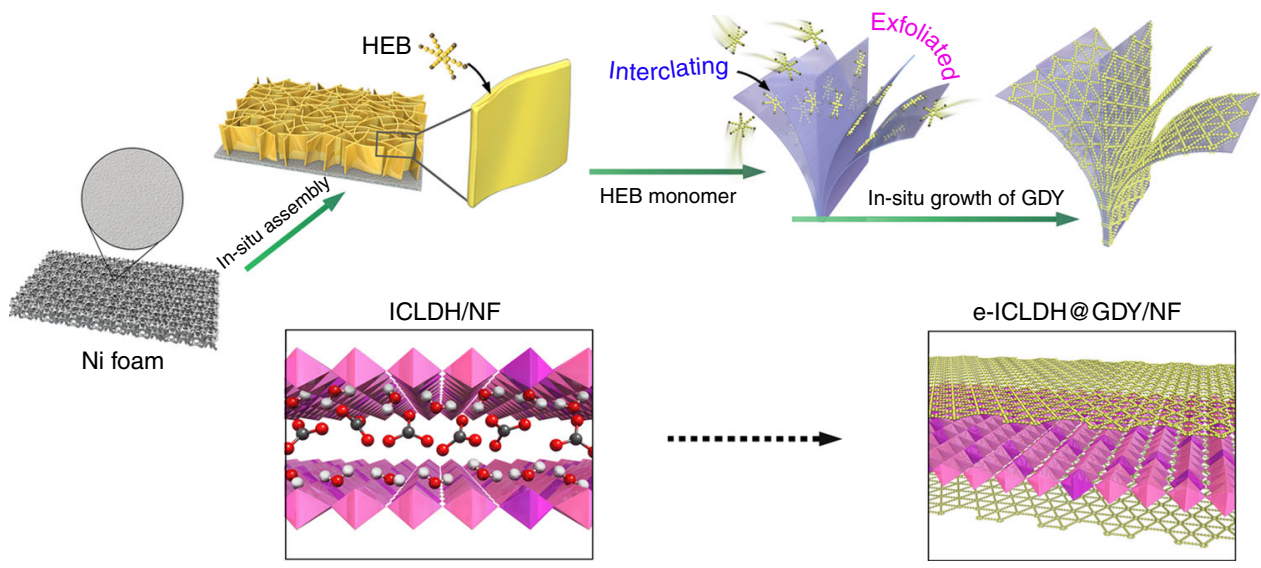

Fig. 1 Schematic representation of the synthetic strategy for the preparation of e-ICLDH@GDY/NF structures
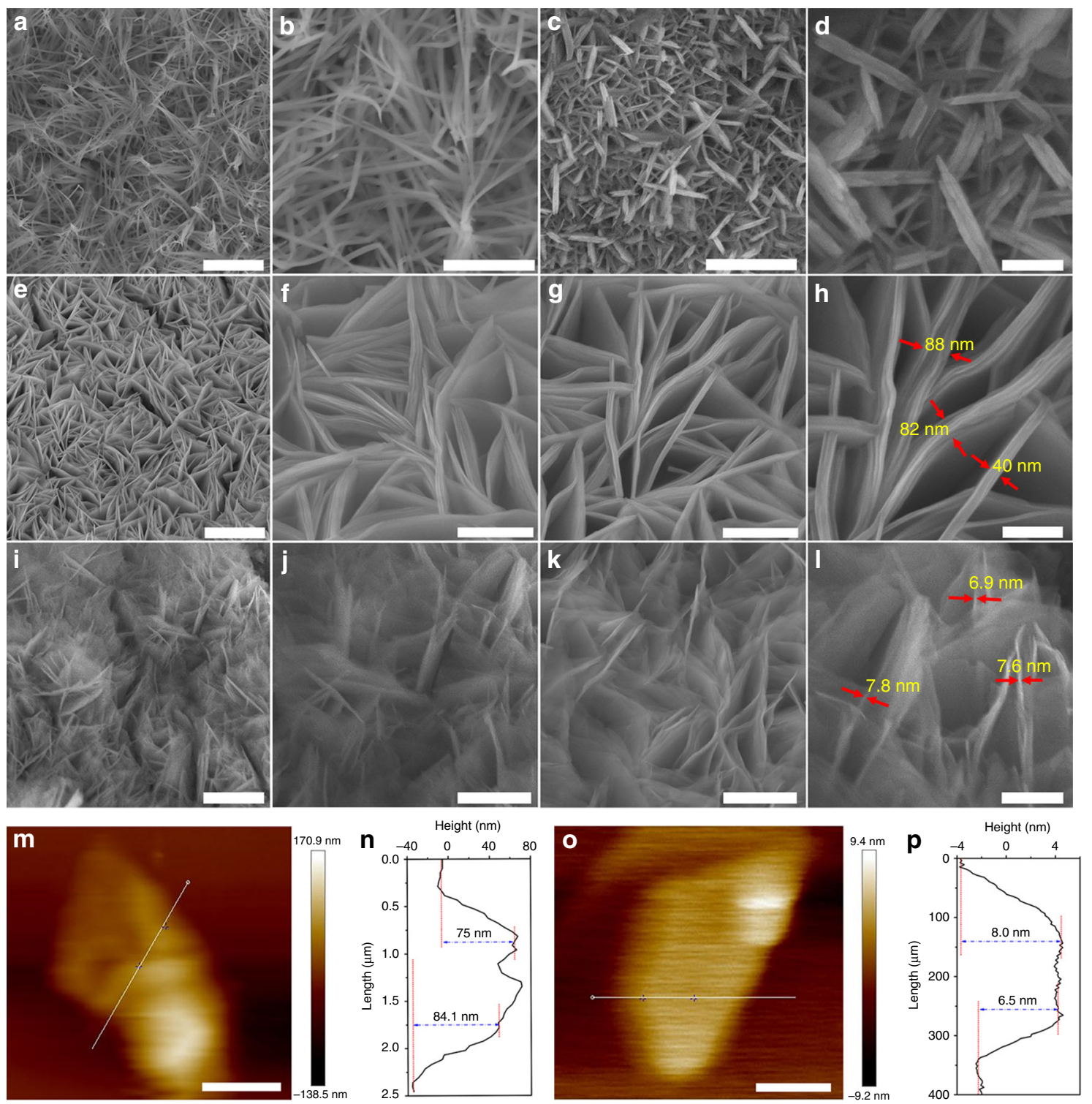

Fig. 2 SEM images of LDH/NF samples. a Low-magnification and b high-magnification SEM images of CLDH/NF. Scale bars: a $2 \mu$ m; b 500 nm. c Lowmagnification and $\mathbf{d}$ high-magnification SEM images of ILDH/NF (recorded at different areas). Scale bars: $\mathbf{c} 1 \mu \mathrm{m} ; \mathbf{b} 200 \mathrm{~nm}$. e-I SEM images of e-h pristine ICLDH/NF and i-I e-LDH@GDY/NF (recorded at different areas). Scale bars: e $2 \mu \mathrm{m} ; \mathbf{f}, \mathbf{g}, \mathbf{j}, \mathbf{k} 500 \mathrm{~nm} ; \mathbf{i} 1 \mu \mathrm{m} ; \mathbf{h}, \mathbf{I} 200 \mathrm{~nm}$. AFM images and height profiles of $\mathbf{m}, \mathbf{n}$ ICLDH nanosheets and $\mathbf{0}, \mathbf{p}$ e-LDH@GDY nanosheet, respectively. Scale bars: $\mathbf{m} 1 \mu \mathrm{m} ; \mathbf{0} 150 \mathrm{~nm}$ 


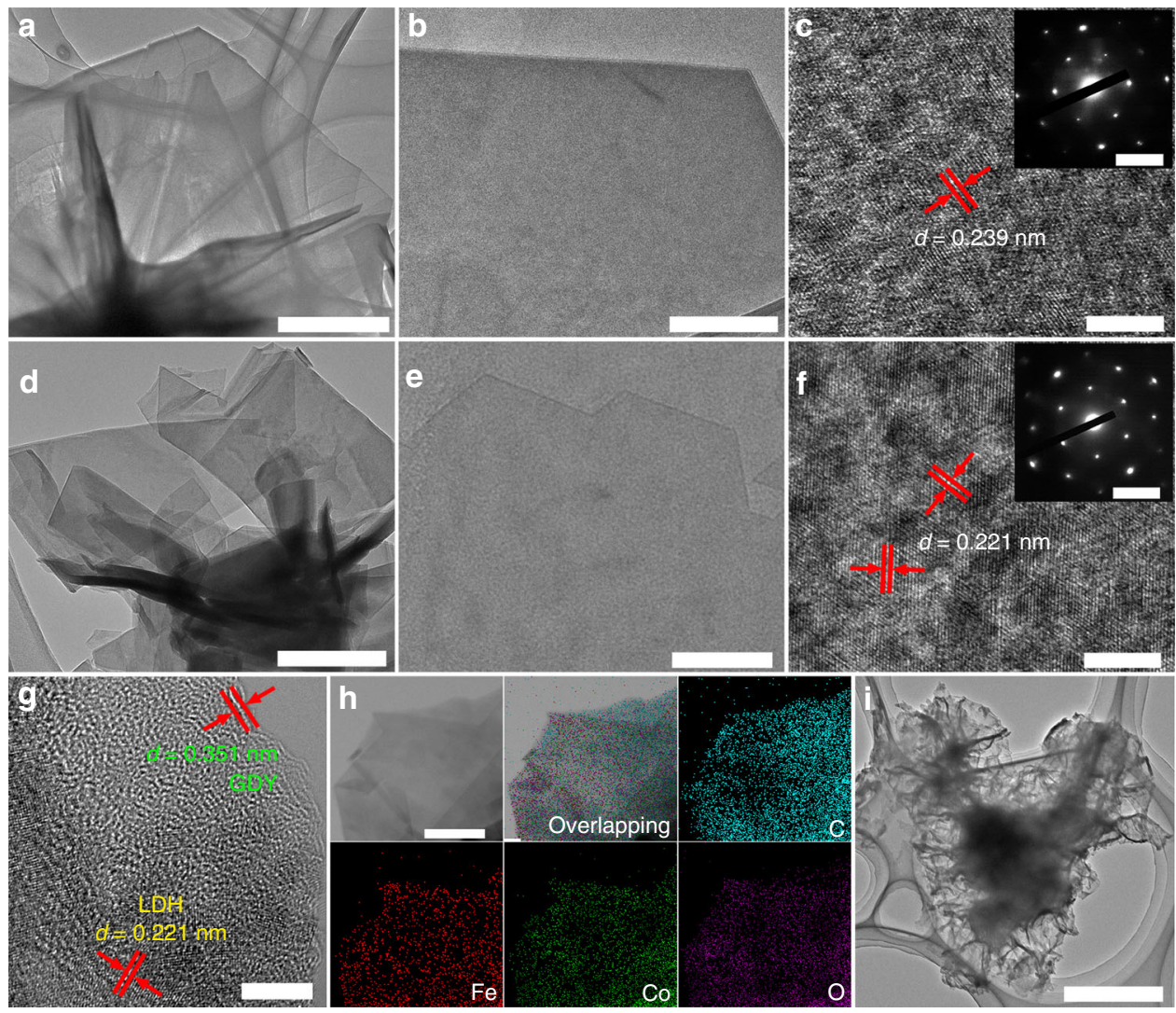

Fig. 3 TEM characterization of electrocatalyst samples. a Low-magnification, $\mathbf{b}$ high-magnification, and $\mathbf{c}$ high-resolution TEM images and corresponding selected-area electron diffraction pattern (SAED, inset to c of pure ICLDH nanosheets. Scale bars: a $1 \mu$ m; b $300 \mathrm{~nm}$; c $5 \mathrm{~nm}$; inset of c, $51 / \mathrm{nm}$. d Lowmagnification, e high-magnification, and $\mathbf{f}$, g high-resolution TEM images of e-ICLDH@GDY nanosheets (inset to f SAED pattern of e-ICLDH@GDY). Scale bars: $\mathbf{d} 200 \mathrm{~nm}$; e $100 \mathrm{~nm}$; $\mathbf{f}$ and $\mathbf{g} 5 \mathrm{~nm}$; inset of $\mathbf{f} 51 / \mathrm{nm}$. h Typical scanning TEM and corresponding elemental mapping images of C, Fe, Co, and $\mathrm{O}$ atoms in the e-ICLDH@GDY nanosheets. Scale bars: $\mathbf{h} 100 \mathrm{~nm}$. i TEM image of the GDY coatings after removal of the ICLDH from the sample.

Scale bars: i $500 \mathrm{~nm}$

for synthesizing e-ICLDH@GDY/NF. SEM images exhibited no any changes in morphology before and after the treatments by pyridine or TMEDA (Supplementary Figs. 1 and 2). These observations indicated the exfoliation of the bulk LDHs.

Compared with the pristine ICLDH nanosheets (Fig. 3a, b), the TEM images of the e-ICLDH@GDY nanosheets (Fig. 3d, e) exhibited weak contrast, suggesting that they were very thin. The selected-area electron diffraction (SAED) patterns of the pristine ICLDH (inset to Fig. 3c) and e-ICLDH@GDY nanosheets (inset to Fig. 3f) both featured hexagonally arranged spots, revealing that the single-crystalline nature of the LDHs remained after their coating with the GDY layer. High-resolution TEM (HRTEM) images revealed that the lattice spacing of the pristine $\mathrm{LDH}$ decreased from $0.239 \mathrm{~nm}$ (Fig. 3c) to $0.221 \mathrm{~nm}$ (Fig. 3f) after growth of the GDY layers, suggesting strong interactions between the GDY and the ICLDH layers in the e-ICLDH@GDY. A fringe distance of $0.351 \mathrm{~nm}$ and a lattice spacing of $0.221 \mathrm{~nm}$, corresponding to the GDY and ICLDH layers, respectively, are also be clearly evident in Fig. 3g. Supplementary Fig. 3 shows the HRTEM image of the lateral standing e-ICLDH@GDY nanosheet. The thickness of GDY and LDH are determined to be about $1.2 \mathrm{~nm}$ and 5.1-6.5 nm, respectively, which is in accordance with the SEM (Fig. 2i) and AFM imaging (Fig. 2o, p) results. Furthermore, scanning TEM (STEM) and energy dispersive X-ray spectroscopy (EDS) mapping images demonstrated the homogeneous distribution of $\mathrm{Fe}, \mathrm{Co}, \mathrm{C}$, and $\mathrm{O}$ elements within the whole nanosheet structure (Fig. $3 \mathrm{~h}$ and Supplementary Fig. 4). The EDS pattern (Supplementary Fig. 5) revealed that the nanosheets contained only the elements $\mathrm{Fe}, \mathrm{Co}$, $\mathrm{C}$, and $\mathrm{O}$. Moreover, after treatment with concentrated nitric acid, the GDY coatings delaminated from the pristine ICLDHs and retained the basic nanosheet morphology of the $\mathrm{LDH}$ nanosheet array (Fig. 3i, Supplementary Fig. 6). These observations confirmed the successful growth of GDY layers on the LDH and the intimate contact between the layers of single-crystallized LDHs and GDY.

We employed Fourier transform infrared (FTIR) spectroscopy to characterize our electrocatalysts. For the e-ICLDH@GDY/NF and pristine ICLDH/NF samples, we attribute the broad intense bands between 3100 and $3600 \mathrm{~cm}^{-1}$ to stretching of their $\mathrm{OH}$ groups (Fig. $4 \mathrm{a}-\mathrm{c}$ ). As revealed in Fig. $4 \mathrm{~b}$, the band at $1503 \mathrm{~cm}^{-1}$, which generally corresponds to the stretching mode of interlayer carbonate species, is present only in the spectrum of pristine ICLHD/NF ${ }^{32}$. In addition, in the spectrum of e-ICLDH@GDY/ $\mathrm{NF}$, the bands at 1450 and $1587 \mathrm{~cm}^{-1}$ represent skeletal vibrations of the aromatic ring, while the band at $2103 \mathrm{~cm}^{-1}$ corresponds to the stretching of $\mathrm{C} \equiv \mathrm{C}$ bonds (Fig. 4c) ${ }^{16}$. We used X-ray photoelectron spectroscopy (XPS) to obtain information regarding the elemental compositions and chemical states of our assynthesized samples. The XPS survey spectrum of the eICLDH@GDY nanosheets revealed that only the elements Fe, $\mathrm{Co}, \mathrm{C}$, and $\mathrm{O}$ were present in the nanosheets (Fig. 4d). Compared with the XPS survey spectrum of pristine ICLDH, the absence of the peak in the range $288.0-290.2 \mathrm{eV}$ implied the thorough removal of $\mathrm{CO}_{3}{ }^{2-}$ ions from the e-ICLDH structure after incorporation with GDY (Fig. 4e). Figure $4 \mathrm{f}$ presents the $\mathrm{O} 1 \mathrm{~s}$ 

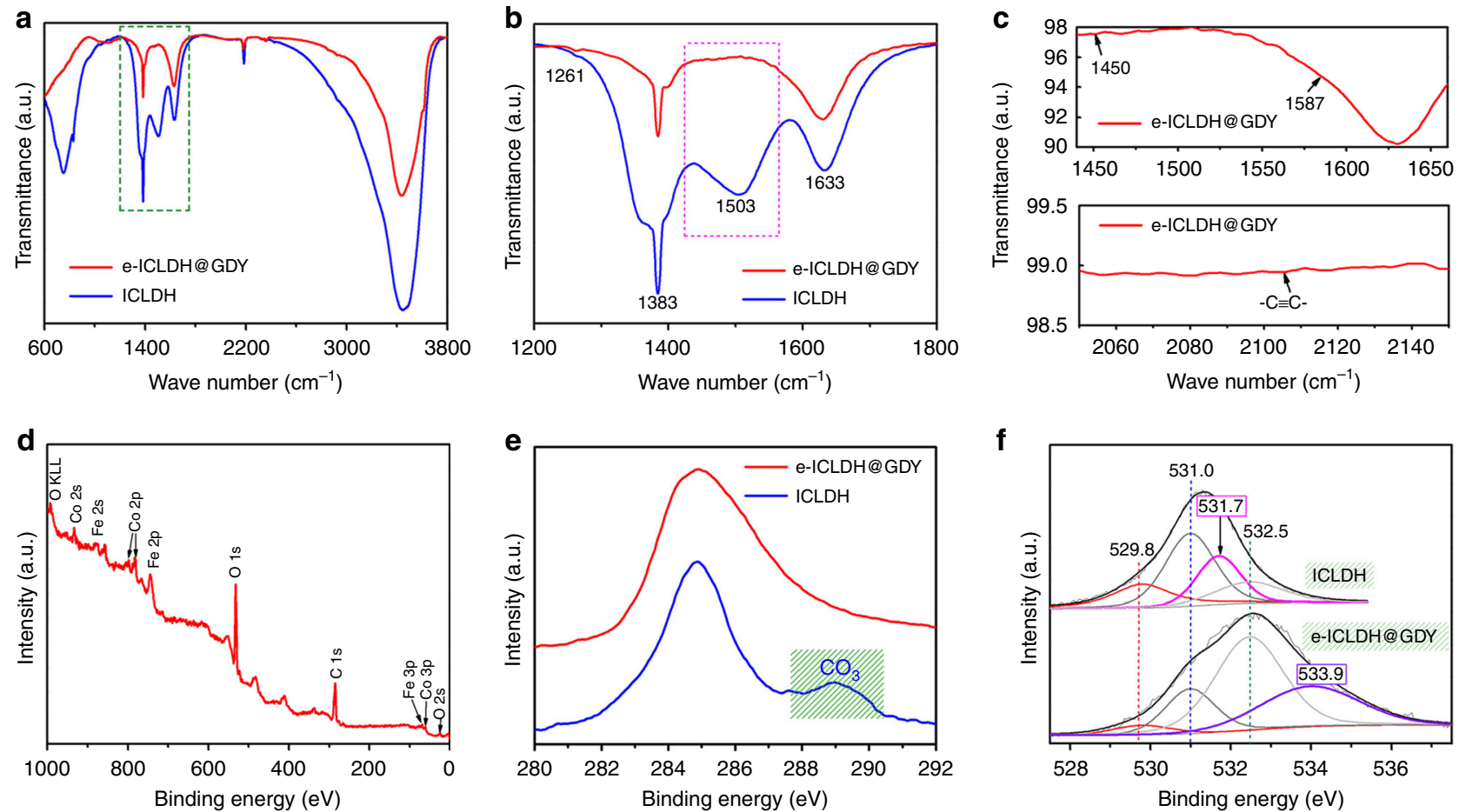

Fig. 4 Structural characterization of electrocatalysts. a FTIR spectra of the e-ICLDH@GDY/NF (red line) and pristine ICLDH/NF (blue line) structures. b Enlarged image of the selected area of the FTIR spectrum in a. c Selective enlargement of the FTIR spectra of e-ICLDH@GDY/NF. d XPS survey spectrum of e-ICLDH@GDY/NF. e, f Core-level XPS spectra of the e C 1s and $\mathbf{f} O$ 1s binding energies of the e-ICLDH@GDY/NF and pristine ICLDH/NF electrocatalysts

XPS spectra of the pristine ICLDH and e-ICLDH@GDY samples. For the pristine ICLDH, we deconvoluted the $\mathrm{O} 1 \mathrm{~s}$ spectrum into four sub-peaks at 529.8, 531.0, 531.7, and $532.5 \mathrm{eV}$, which we ascribe to lattice oxygen $\left(\mathrm{O}^{2-}\right)$, metal-oxygen bonding $(\mathrm{M}-\mathrm{O}-\mathrm{M})$, adsorbed oxygen in the form of $\mathrm{CO}_{3}^{2-}$, and $\mathrm{OH}$ groups, respectively ${ }^{33}$. In the spectrum of e-ICLDH@GDY, four peaks were present, located at 529.8, 531.0, 532.5, and $533.9 \mathrm{eV}$ (Fig. 4f). We infer that the peak corresponding to $\mathrm{CO}_{3}{ }^{2-}$ ions $(533.9 \mathrm{eV})$ disappeared, while a peak for $\mathrm{C}-\mathrm{O}$ bonds $(533.9 \mathrm{eV})$ appeared. The $\mathrm{C}-\mathrm{O}$ species originated from in situ growth of GDY on the LDH surface. These spectra confirmed the removal of the interlayer anions $\left(\mathrm{CO}_{3}{ }^{2-}\right)$ from the $\mathrm{LDH}$ and the formation of the e-ICLDH@GDY structure.

Theoretical studies. We performed theoretical calculations of the impact of GDY on the electronic structure of the electrocatalyst. The configurations of GDY (Fig. 5a), ICLDH (Fig. 5b, Supplementary Fig. 7 and Supplementary Methods) and ICLDH@GDY (Fig. 5c) were firstly optimized. ICLDH@GDY featured smaller lattice parameters $(a=9.34 \AA ; b=9.36 \AA)$ than did the pristine $\operatorname{GDY}(a=b=9.46 \AA)$, implying that the incorporation of LDH and GDY led to shrinkage of the GDY structure-possibly a positive influence on the catalytic activity. Furthermore, our calculations indicated that 0.80 lel was transferred in total from ICLDH to the GDY layer, indicative of strong electronic interactions between the GDY and the LDHs (Fig. 5d). This theoretical prediction was confirmed in XPS (Fig. 5e, f) and Raman (Fig. 5g) spectra. The binding energies of both $\mathrm{Fe} 2 \mathrm{p}_{3 / 2}$ (Fig. 5e) and Co $2 \mathrm{p}_{3 / 2}$ (Fig. $5 \mathrm{f}$ ) in the pristine ICLDH were increased by $2.2 \mathrm{eV}$ (from 712.2 to $714.4 \mathrm{eV}$ ) and $3.0 \mathrm{eV}$ (from 780.6 to $783.6 \mathrm{eV}$ ) after in situ growth of GDY, indicating the presence of strong electronic interactions between GDY and the metallic species in the LDHs. In the Raman spectrum of e-ICLDH@GDY (Fig. 5h), the peaks corresponding to the metal-OH stretching vibration modes (at 448.4 and $528.5 \mathrm{~cm}^{-1}$ ), the $\mathrm{D}$ band $\left(1398.4 \mathrm{~cm}^{-1}\right.$ ), the $\mathrm{G}$ band $\left(1576.9 \mathrm{~cm}^{-1}\right)$, and the vibrations of conjugated $-\mathrm{C} \equiv \mathrm{C}-$ units (1920.6 and $2176.4 \mathrm{~cm}^{-1}$ ) were shifted significantly when compared with the spectra of pristine ICLDH and GDY. We expected such associated electronic interactions between GDY and LDH to be very beneficial for the electrocatalytic performance. For the OER in alkaline electrolyte (Supplementary Fig. 8 ), the ${ }^{*} \mathrm{O}$-to${ }^{*} \mathrm{OOH}$ conversion step is believed to be rate-determining ${ }^{34}$. Electrostatic interactions at the electrocatalyst interface afford a driving force for the adsorption of ${ }^{*} \mathrm{O}$, in turn accelerating the conversion of ${ }^{*} \mathrm{O}$ to ${ }^{*} \mathrm{OOH}$. A greater density of electrons at the electrocatalyst surface should be of more help for the formation of ${ }^{*} \mathrm{OOH}$ and thus, enhance the OER activity ${ }^{34-36}$. As demonstrated experimentally and theoretically above (Fig. 5), the obvious electron transfer from LDH to GDY in e-ICLDH@GDY should, therefore, favor the formation of ${ }^{*} \mathrm{OOH}$ species with a smaller free energy of $0.54 \mathrm{eV}$ than that of pure GDY $(0.93 \mathrm{eV}$, Fig. $5 \mathrm{~h})$ and benefit the OER activity. In addition, it has been reported that electrons transferred from adjacent metal species (e.g., $\mathrm{Fe}^{37}$, $\mathrm{Ni}^{37}, \mathrm{Mo}^{29}$ ) to GDY can facilitate both the adsorption (the ratedetermining step) and desorption of $\mathrm{H}$ atoms, leading to greater HER activity.

Here we further gained some insightful interpretations on the high alkaline HER performance on the HER by DFT calculations (Fig. 5, Supplementary Fig. 9). The projected partial density of states (PDOSs) show the related p-and d-band distributions for the nearest interfacing GDY and LDH layers. The overall 3d-band center of ICLDH is high next to the Fermi level $\left(\mathrm{E}_{\mathrm{F}}\right)$, which is responsible for activating the initial adsorptions of $\mathrm{O}$-species from alkaline condition. The partial intrinsic overlaps of $\mathrm{p}$ - and $\mathrm{d}$-bands demonstrate a weak inter-layer bonding. This indicates a rather long-ranged interacting interfacing layer with large distance for $\mathrm{H}_{2} \mathrm{O}$ molecule easily passing through, without obviously impacting the catalyst interface (Fig. 5i). Details on the 3d-bands of Co and Fe sites from LDH interfacing layer show 
a
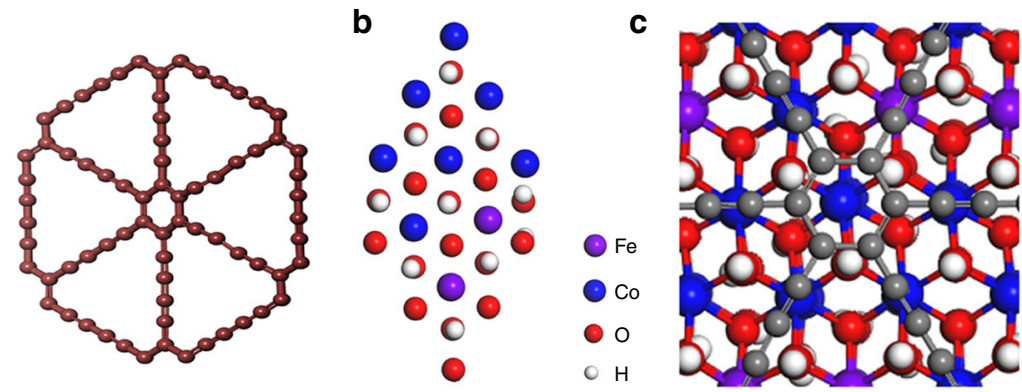

e

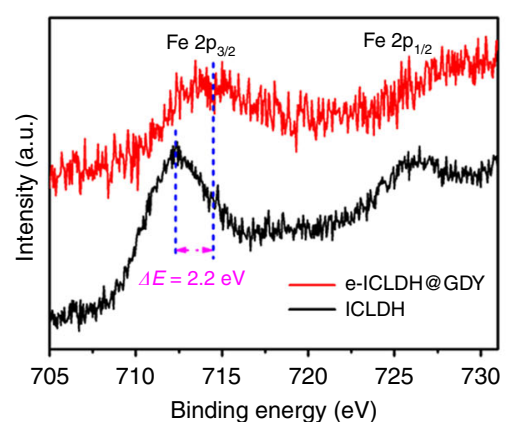

f

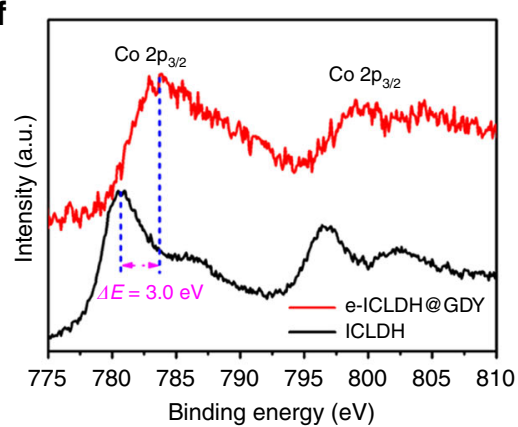

h

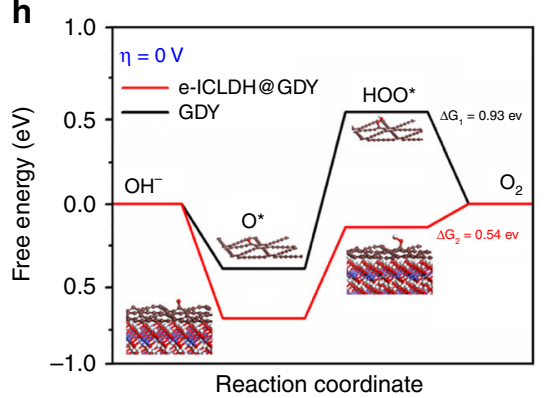

\section{i}
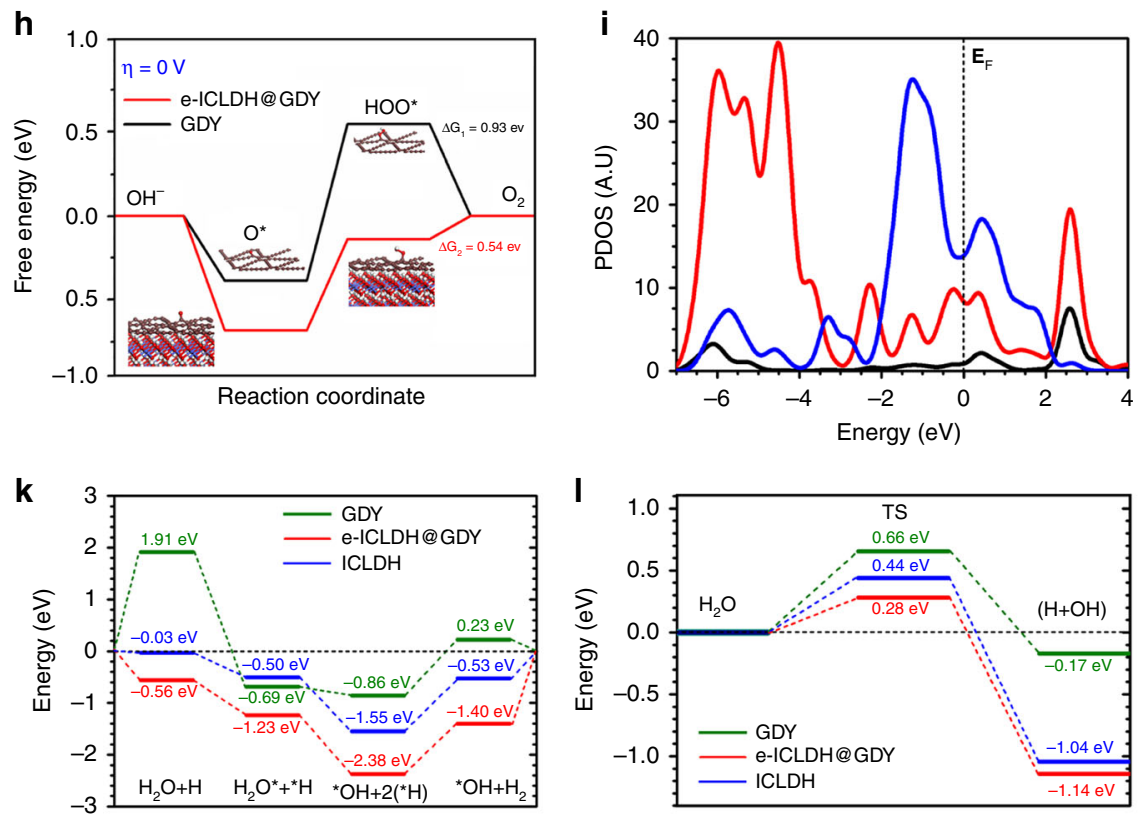

I

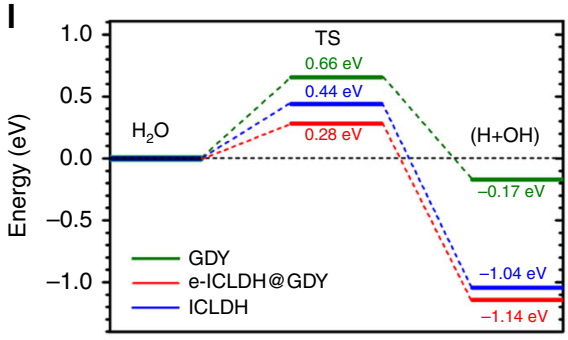

d

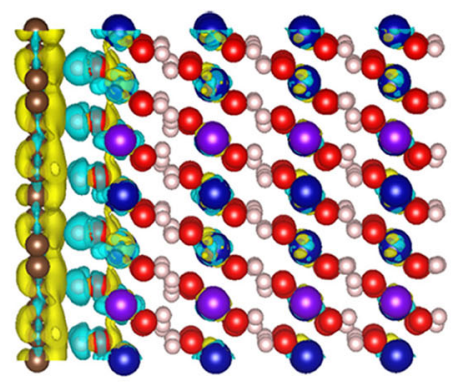

$\mathbf{g}$

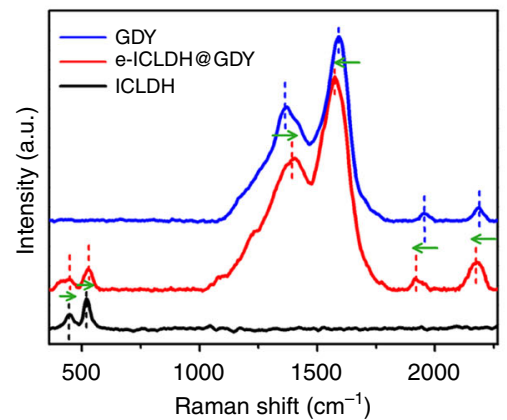

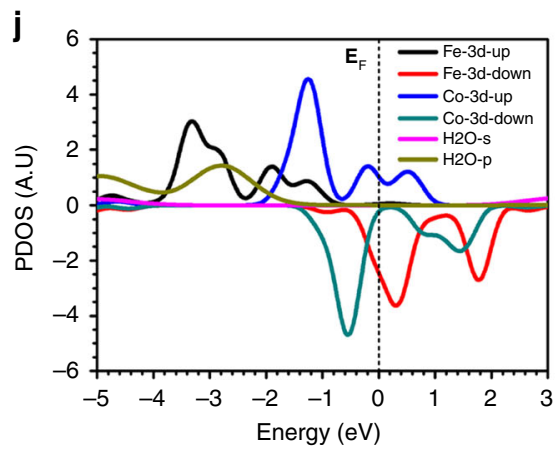

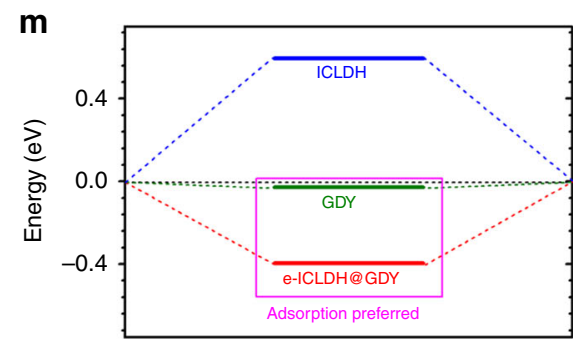

n
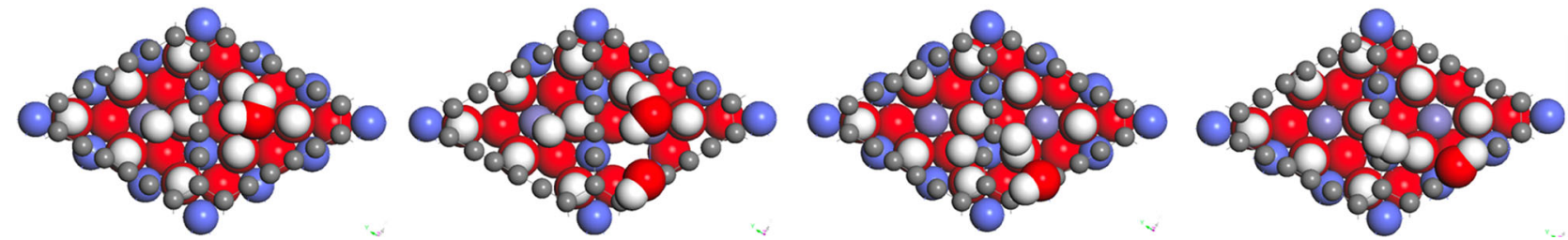

Fig. 5 Theoretical calculations and structural analysis of the electrocatalysts. The stable configurations of a GDY, b ICLDH and c ICLDH@GDY.d Charge density difference for the stable configuration of ICLDH@GDY.e Fe 2p and f Co 2p core-level XPS spectra of the e-ICLDH@GDY/NF structure. $\mathbf{g}$ Raman spectra of GDY, ICLDH, and e-ICLDH@GDY; arrows indicate the directions of the shifts of the Raman spectral signals relative to those of e-ICLDH@GDY. $\mathbf{h}$ The free energy changes for the formation of $\mathrm{OOH}^{\star}$ and corresponding stable structures of GDY $\left(\Delta \mathrm{G}_{1}\right)$ and e-ICLDH@GDY $\left(\Delta \mathrm{G}_{2}\right)$. i PDOSs of the $3 \mathrm{~d}$ and $2 \mathrm{p}$ bands of interfaced system containing GDY and ICLDH layers. $\mathbf{j}$ PDOSs of Fe-3d, Co-3d, $\mathrm{H}_{2} \mathrm{O}-\mathrm{s}$ and $\mathrm{H}_{2} \mathrm{O}-\mathrm{p}$ bands near the interface region. k Energetic pathway of HER under alkaline conditions for e-ICLDH@GDY, ICLDH, and GDY, respectively. I Comparison on the transition state barrier for $\mathrm{H}_{2} \mathrm{O}$-splitting among three systems. $\mathbf{m} \mathrm{H}$-chemisorption of these three systems. n Structural evolution path for alkaline HER within e-ICLDH@GDY interface system 
the contrast in $3 \mathrm{~d}$-band centers, which potentially denotes a dynamically self-activated electronic activity of redox. The Co-3d bands with majority-spin is pinned at the $\mathrm{E}_{\mathrm{F}}$ and locates $1.4 \mathrm{eV}$ higher than one of $\mathrm{Fe}-3 \mathrm{~d}$ band. This trends show $\mathrm{Fe}-3 \mathrm{~d}$ acts as electron-rich center while the Co-3d band plays as electrondepletion channel. However, their d-d overlapping is rather weak with few overlaps in PDOS. This indicates the highly effective electron-transfer occurs between the inter-layers instead of intralayer ionic sites. The $\mathrm{O}-2 \mathrm{p}$ band from $\mathrm{H}_{2} \mathrm{O}$ exhibits a large overlapping across the $\mathrm{Fe}-3 \mathrm{~d}$ band confirming the Fe-site acts as dominant role for $\mathrm{H}_{2} \mathrm{O}$ initial adsorption within interlayer regions (Fig. 5j). We move onto the energetic pathway of the alkaline HER for this interface system ${ }^{38,39}$. Overall, the eICLDH@GDY system performs the most energetic favorable path with lowest adsorption $(-0.56 \mathrm{eV})$ and preferred exothermic reaction heat for $\mathrm{H}_{2}(-1.40 \mathrm{eV})$. In the contrast, the GDY exhibits the highest barrier for initial $\mathrm{H}_{2} \mathrm{O}$ adsorption $(1.92 \mathrm{eV})$ and an endothermic reaction $(0.23 \mathrm{eV})$ for HER. The capabilities of water-splitting demonstrate the preference as e-ICLDH@GDY > ICLDH > GDY, respectively (Fig. 5k). We also compared the intermediating $\mathrm{H}_{2} \mathrm{O}$-spliting and the transition state barriers are $0.28 \mathrm{eV}$ (e-ICLDH@GDY), $0.44 \mathrm{eV}$ (ICLDH), and $0.65 \mathrm{eV}$ (GDY), respectively (Fig. 5l). Meanwhile, the chemisorption energy of HER has been also illustrated. Among of them, the e-ICLDH@GDY indicates even more energetic favorable trend for HER compared with pristine-GDY, while the LDH meets the unflavored uphill for $\mathrm{H}$-adsorption (Fig. $5 \mathrm{~m}$ ). From the local structures (Fig. 5n), the stable adsorbed $\mathrm{H}$ is bonding with $\mathrm{C} 2$ site at the GDY layer and the $\mathrm{H}_{2} \mathrm{O}$ location will pass through the GDY locating near Fe-site on the ICLDH surface. The splitting $\mathrm{H}_{2} \mathrm{O}$ process occurs between the $\mathrm{C} 2$ site and Fe-site from $\mathrm{LDH}$ layer. The two closely adsorbed $\mathrm{H}$ exhibit a favorable trend for combination in structural configuration for potentially efficient $\mathrm{H}_{2}$ generation. With assistance of $\mathrm{C} 2$ site, the leaving group $\left(\mathrm{OH}^{-}\right)$can be stably located on the surface of GDY. Accordingly, we suspected that the presence of the associated electronic interactions between GDY and ICLDH might improve the intrinsic electrocatalytic HER activity of our e-LDH@GDY/ NF electrocatalyst.

Electrocatalytic OER catalysis. As a proof-of-concept application, we first applied the e-ICLDH@GDY/NF structure as an OER catalyst in $1.0 \mathrm{M}$ aqueous $\mathrm{KOH}\left(4 \mathrm{OH}^{-} \rightarrow \mathrm{O}_{2}+4 \mathrm{e}^{-}+2 \mathrm{H}_{2} \mathrm{O}\right)$ using a typical three-electrode system operated at $1 \mathrm{mV} \mathrm{s}^{-1}$. We used cyclic voltammetry (CV) to measure the OER activities. As shown in Fig. 6a and Supplementary Fig. 10, the e-ICLDH@GDY/ NF exhibited the best OER activity with the smallest overpotentials of $216,238,249,275$, and $278 \mathrm{mV}$ at current densities (j) of 10 , $50,100,500$, and $1000 \mathrm{~mA} \mathrm{~cm}^{-2}$, respectively-values that are comparable to or even better than those of previously reported LDH-related OER catalysts (Supplementary Table 1), including $(\mathrm{Ni}, \mathrm{Fe}) \mathrm{OOH}\left(289 \mathrm{mV} \text { at } 10 \mathrm{~mA} \mathrm{~cm}^{-2}\right)^{40}, \mathrm{CoO}_{\mathrm{x}} @ \mathrm{CN}(260 \mathrm{mV}$ at $\left.10 \mathrm{~mA} \mathrm{~cm}^{-2}\right)^{41}$, 3DGN/CoAl-NS $\left(252 \mathrm{mV} \text { at } 10 \mathrm{~mA} \mathrm{~cm}^{-2}\right)^{11}$, and $\mathrm{Co}_{1} \mathrm{Mn}_{1} \mathrm{CH}\left(322 \mathrm{mV} \text { at } 50 \mathrm{~mA} \mathrm{~cm}^{-2}\right)^{10}$. (Supplementary Fig. 11) The performance of e-ICLDH@GDY/NF revealed a small Tafel slope of $43.6 \mathrm{mV} \mathrm{dec}{ }^{-1}$ (Fig. 6b, Supplementary Fig. 11), much smaller than those of $\mathrm{RuO}_{2}(76.4 \mathrm{mV} \mathrm{dec}-1)$ and most of the reported precious and nonprecious electrocatalysts (Supplementary Table 1). From the OER results (Supplementary Fig. 10), we can see there are two major factors can make the enhanced activity. One is GDY incorporation, and another is Fe introduction into CLDH. From the polarization curves, obviously, incorporation of Fe has greater effects on the activity. As shown in Supplementary Fig. 12, the CVs for pure CLDH/NF and CLDH@GDY/NF exhibit two pronounced oxidation peaks at 1.28 and $1.36 \mathrm{~V}$ corresponding to the oxidation of $\mathrm{Co}^{2+}$ to $\mathrm{Co}^{3+}$ and the oxidation of $\mathrm{Co}^{3+}$ to $\mathrm{Co}^{4+}$, respectively ${ }^{42-44}$. For pure ILDH, the oxidation peak was observed at round $1.41 \mathrm{~V}^{45}$. The CV of e-ICLDH@GDY/NF shows only one broad anodic peak at around $1.25 \mathrm{~V}$ and a cathodic peak at around $1.16 \mathrm{~V}$ (Supplementary Fig. 12d). The redox behavior of the e-ICLDH@GDY/NF sample is mainly attributed to the $\mathrm{Co}^{2+}$ / $\mathrm{Co}^{3+}$ and $\mathrm{Co}^{3+} / \mathrm{Co}^{4+}$ redox pairs and contribution from iron $^{34,43,46,47}$. Such high charge densities would be particularly beneficial for the improvement of the OER catalytic activity ${ }^{34,43}$ Theoretical studies showed the effects of Fe incorporation (Supplementary Fig. 13 and Supplementary Methods). After the configuration optimization, the calculated binding energies $\left(\mathrm{E}_{\mathrm{b}}\right)$ are $-2.01 \mathrm{eV}$ and $-2.26 \mathrm{eV}$ for CLDH@GDY and ICLDH@GDY, respectively, which shows that the incorporation of $\mathrm{Fe}$ atoms contribute to the binding between GDY and LDH. Besides, we have also examined the charge transfer between the $\mathrm{LDH}$ and GDY. Compared with pure CLDH $(0.70 \mathrm{lel})$, a more electron in total $(0.80|\mathrm{e}|)$ was transferred from ICLDH to GDY, indicating that the incorporation of $\mathrm{Fe}$ can further enhance the electron transfer, which would be helpful for improving the overall catalytic activity. The long-term stability through continuous CV sweep and chronopotentiometric measurements were carried out in $1.0 \mathrm{M}$ $\mathrm{KOH}$. Impressively, there was only an increase of $8 \mathrm{mV}$ in the overpotential at $50 \mathrm{~mA} \mathrm{~cm}^{-2}$ after 47000 cycles (Fig. 6c), with its morphology retained (Supplementary Fig. 14), indicating excellent long-term durability. In a control experiment, the pristine ICLDH/ NF exhibited a significant decrease (ca. 39\%) in catalytic current density at $1.6 \mathrm{~V}$ vs. RHE after only 3000 cycles. The Faradaic efficiency of e-ICLDH@GDY/NF for OER is close to $100 \%$ $(97.98 \pm 0.81 \%$, Supplementary Fig. 15). Thus, the presence of GDY played an important role in improving the OER activity and stability.

We examined the relationship between temperature relation and electrocatalytic activity to determine the kinetic barriers for the OER (Fig. 6d) ${ }^{48}$. At elevated temperatures, the OER proceeded more rapidly. The overpotentials required to deliver a current density of $10 \mathrm{~mA} \mathrm{~cm}^{-2}$ decreased from $216 \mathrm{mV}$ (at $25^{\circ} \mathrm{C}$ ) to $191 \mathrm{mV}$ (at $50{ }^{\circ} \mathrm{C}$ ), $175 \mathrm{mV}$ (at $60{ }^{\circ} \mathrm{C}$ ), and $158 \mathrm{mV}$ (at $70^{\circ} \mathrm{C}$ ). As displayed in Fig. 6e, the Tafel slopes decreased from $43.6 \mathrm{mV}$

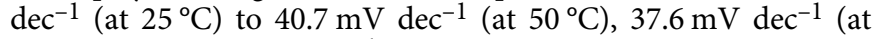

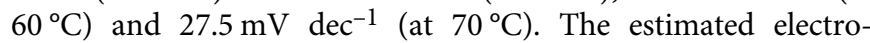
chemical activation energies $\left(\mathrm{E}_{\mathrm{a}}\right)$ of the OER can be extracted from the Arrhenius plots (Fig. 6f), according to the Eq. (1):

$$
\mathrm{Ea}=-2.303 \mathrm{R}\left[\frac{\partial \log (j)}{\partial\left(\frac{1}{\mathrm{~T}}\right)}\right]
$$

where $R$ is the universal gas constant and $T$ is absolute temperature. We constructed Arrhenius plots based on the current densities recorded at different potentials. As the potentials increased from 1.43 to 1.44 to $1.45 \mathrm{~V}$ (v. RHE), the activation energies decreased from 100.9 to 91.52 to $82.33 \mathrm{~kJ} \mathrm{~mol}^{-1}$, respectively, indicating the potential-determining step of our electrocatalyst.

Electrocatalytic HER catalysis. We investigated the HER performance of our structures $\left(2 \mathrm{H}_{2} \mathrm{O}+2 \mathrm{e}^{-} \rightarrow \mathrm{H}_{2}+2 \mathrm{OH}^{-}\right)$in $1.0 \mathrm{M}$ $\mathrm{KOH}$ at $1 \mathrm{mV} \mathrm{s}^{-1}$. Figure $6 \mathrm{~g}$ and Supplementary Fig. 16 provide the polarization curves after $i R$-compensation of the assynthesized samples. e-ICLDH@GDY/NF exhibited the lowest overpotentials of $43,174,215$, and $256 \mathrm{mV}$ at $10,50,100$, and $1000 \mathrm{~mA} \mathrm{~cm}^{-2}$, respectively. These values are better than those of the pristine ICLDH/NF $\left(202 \mathrm{mV}\right.$ at $\left.10 \mathrm{~mA} \mathrm{~cm}^{-2}\right)$, monometallic LDHs (Fig. 6g), and most previously reported nonprecious HER electrocatalysts in alkaline medium (Supplementary Table 2). Similar to that observed in OER (Supplementary Fig. 10), the HER results (Supplementary Fig. 16) also showed that both the GDY introducing and Fe incorporation into CLDH contributed to the improvement of the HER activity, and $\mathrm{Fe}$ incorporation 

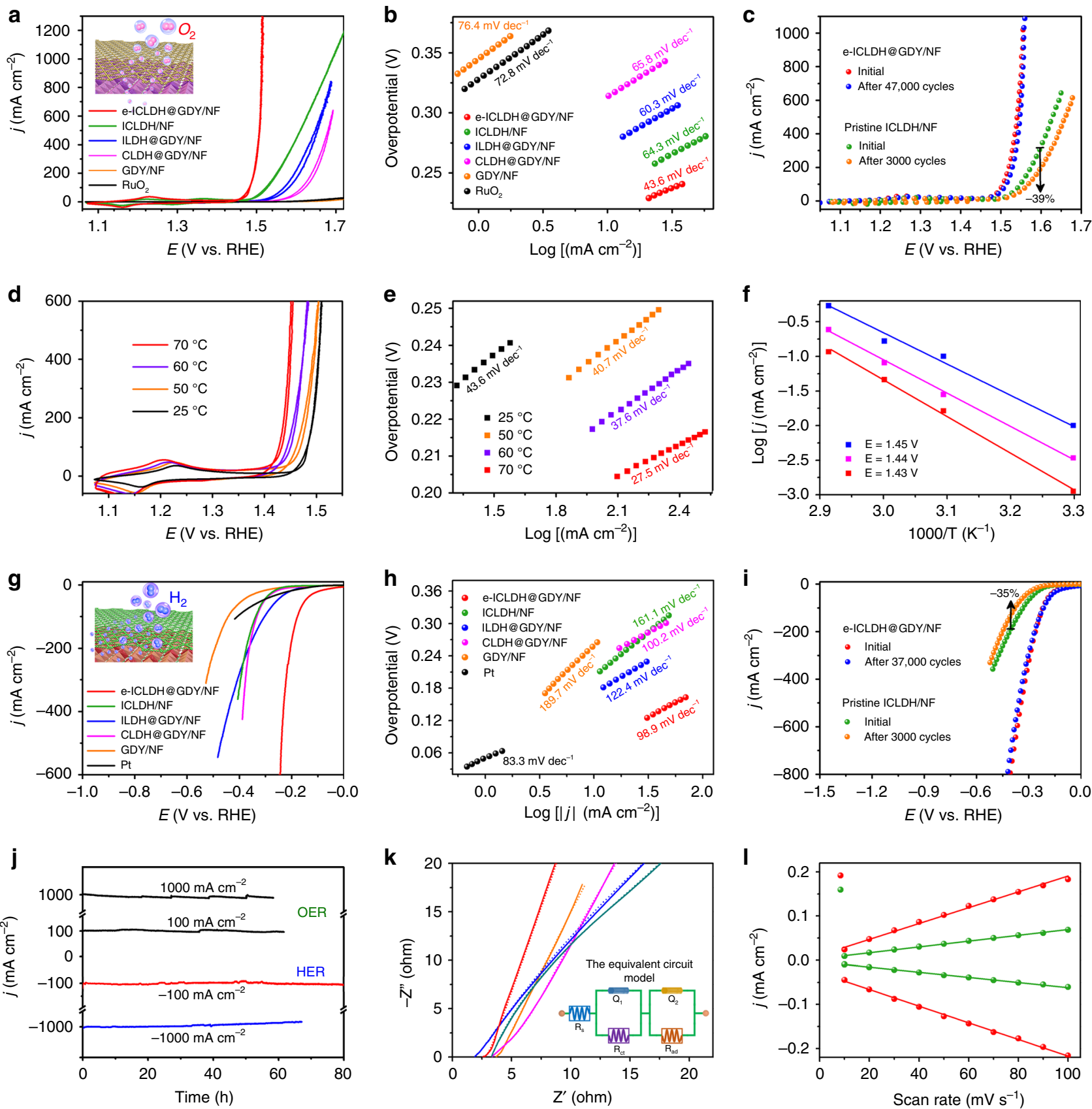

Fig. 6 Electrocatalytic performance of e-ICLDH@GDY/NF. a OER CV curves and $\mathbf{b}$ corresponding Tafel plots of as-synthesized samples. c Polarization curves of e-ICLDH@GDY/NF and pristine ICLDH/NF, recorded before and after performing 47,000 and 3000 cycles, respectively, of the OER. d OER CV curves and e corresponding Tafel plots for e-ICLDH@GDY/NF, recorded at various temperatures. f Arrhenius plots for the OER performed over eICLDH@GDY/NF at various potentials. $\mathbf{g}$ HER polarization curves and $\mathbf{h}$ corresponding Tafel plots for the as-synthesized samples. $\mathbf{i}$ HER polarization curves of e-ICLDH@GDY/NF and pristine ICLDH/NF, recorded before and after 37,000 and 3000 cycles, respectively, of the HER. $\mathbf{j}$ Chronopotentiometric curves of e-ICLDH@GDY/NF recorded at 100 and 1000 mA cm${ }^{-2}$ for both the OER and HER. $\mathbf{k}$ Experimental (solid line) and fitted (dashed line) Nyquist plots for e-ICLDH@GDY/NF (red), ICLDH/NF (green), ILDH/NF (blue), CLDH/NF (magenta), and GDY/NF (orange), respectively; inset: equivalent circuit model. I Capacitive current densities at $0.818 \mathrm{~V}$, plotted with respect to the scan rate, for e-ICLDH@GDY/NF (red) and ICLDH/NF (green)

contributes more. The small Tafel slope value $\left(98.9 \mathrm{mV} \mathrm{dec}^{-1}\right)$, very close to that of $\mathrm{Pt}(83.3 \mathrm{mV} \mathrm{dec}-1)$, confirmed the excellent HER activity of e-ICLDH@GDY/NF (Fig. 6h, Supplementary Fig. 17). We performed continuous cycling tests at $100 \mathrm{mV} \mathrm{s}^{-1}$ to evaluate the durability of e-ICLDH@GDY/NF during the HER process. Only a negligible change in current density occurred after 37,000 continuous CV cycles (Fig. 6i). In contrast, the pure ICLDH/NF exhibited a great decrease in current density, from 186.5 to $120.6 \mathrm{~mA} \mathrm{~cm}^{-2}$, at $-0.4 \mathrm{~V}$ (vs. RHE) after only
3000 cycles. The elemental composition and morphology of e-ICLDH@GDY/NF were both preserved well after the long-term stability tests (Supplementary Fig. 18). We also examined the long-term electrocatalytic stability of the e-ICLDH@GDY/NF structure at current densities of 100 and $1000 \mathrm{~mA} \mathrm{~cm}^{-2}$ for both the OER and HER. During the continuous OER test, the current densities of e-ICLDH@GDY/NF remained nearly constant for 60 and $58 \mathrm{~h}$ at the larger current densities of 100 and $1000 \mathrm{~mA} \mathrm{~cm}^{-2}$, respectively (Fig. 6j). The excellent stability of e-ICLDH@GDY/ 
NF for the HER was further evidenced through constant-current electrolysis tests performed at current densities of $100 \mathrm{~mA} \mathrm{~cm}^{-2}$ for $80 \mathrm{~h}$ (no change in current density) and $1000 \mathrm{~mA} \mathrm{~cm}^{-2}$ for 67 h (Fig. 6j). The Faradaic efficiency of e-ICLDH@GDY/NF for HER was determined to be $98.96 \pm 0.86 \%$ (Supplementary Fig. 19). Taken together, these findings confirmed the outstanding stability and superiority of the e-ICLDH@GDY/NF electrode.

To determine the origin of the excellent activity of e-ICLDH@GDY/NF, we recorded electrochemical impedance spectroscopy (EIS) Nyquist plots (Fig. 6k, Supplementary Fig. 20) to study its charge transfer behavior ${ }^{39}$. We employed an equivalent circuit model (inset to Fig. 6k) featuring solution resistance $\left(R_{\mathrm{s}}\right)$, charge transfer resistance $\left(R_{\mathrm{ct}}\right)$, and adsorption resistance $\left(R_{\mathrm{ad}}\right)$ to fit the Nyquist plots. e-ICLDH@GDY/NF possessed the lowest values of $R_{\mathrm{s}}$ and $R_{\mathrm{ct}}$ (2.46 and $1.42 \Omega$, respectively) among all the electrocatalysts (Supplementary Table 3); in particular, these values were much lower than those of the pristine ICLDH/NF $\left(R_{\mathrm{s}}=3.45 \Omega ; R_{\mathrm{ct}}=21.55 \Omega\right)$ and GDY/ $\mathrm{NF}\left(R_{\mathrm{s}}=3.78 \Omega ; R_{\mathrm{ct}}=23.63 \Omega\right)$, indicating that e-ICLDH@GDY/ NF had the most favorable charge transfer kinetics. In addition, the electrochemical measurements revealed that e-ICLDH@GDY/ NF had a capacitance of $2.0 \mathrm{mF} \mathrm{cm}^{-2}$, approximately 3.3 times that of the pristine ICLDH/NF $\left(0.6 \mathrm{mF} \mathrm{cm}^{-2}\right.$, Fig. $6 \mathrm{l}$,
Supplementary Fig. 21), suggesting that the former had relatively larger electrochemical surface area (ECSA). Turnover frequencies (TOFs) have also been used to assess the information about the intrinsic activity (see Supplementary Methods for details). The e-ICLDH@GDY/NF shows TOF values $8.44 \mathrm{~s}^{-1}$ at $200 \mathrm{mV}$ for HER and $2.34 \mathrm{~s}^{-1}$ at $250 \mathrm{mV}$ for OER, respectively, which are larger than that of pure ICLDH and most of recently reported catalysts (Supplementary Table 4). The improvement in the catalytic activity of e-ICLDH@GDY/NF was presumably due to the incorporation of ICLDH with GDY, which significantly facilitated electron transport and increased the number of electrocatalytic active sites.

Overall water splitting. Considering the outstanding HER and OER performance and stability of the bifunctional e-ICLDH@GDY/NF electrocatalyst, we assembled an alkaline electrolyzer, using e-ICLDH@GDY/NF as both the anode and cathode, in $1.0 \mathrm{M} \mathrm{KOH} \mathrm{(Fig.} \mathrm{7a).} \mathrm{As} \mathrm{shown} \mathrm{in} \mathrm{Fig.} \mathrm{7b,} \mathrm{c,} \mathrm{the}$ e-ICLDH@GDY/NF electrode exhibited the best OWS activity among all samples. For example, the CV curves in Fig. 7c (with $i R$-correction) show that the e-ICLDH@GDY/NF electrode exhibited OWS activity-with small cell voltages of $1.43,1.46$, and
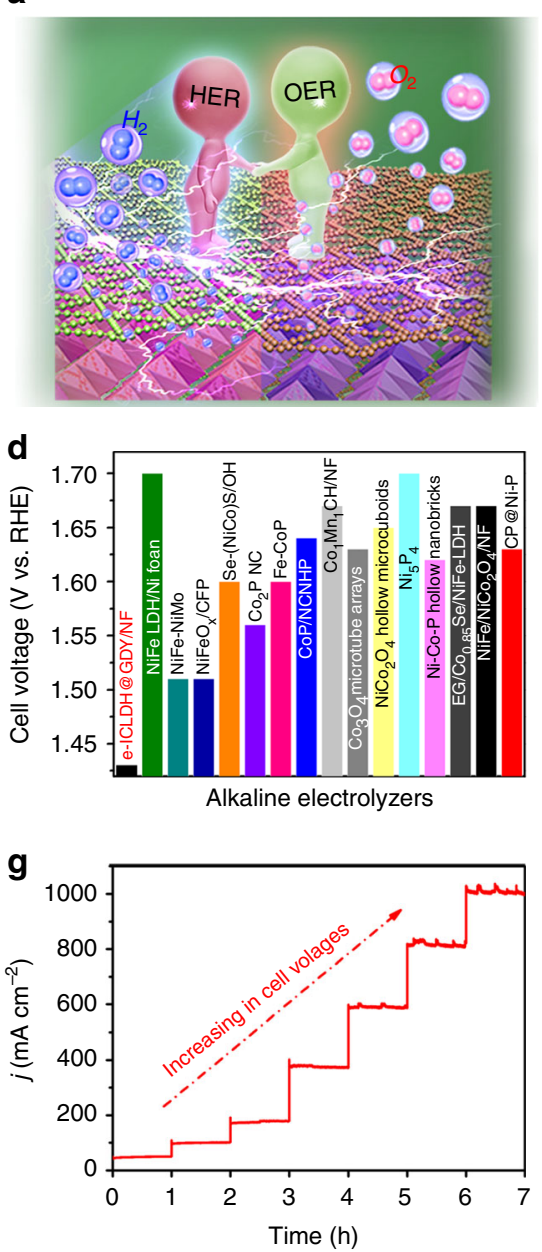

b

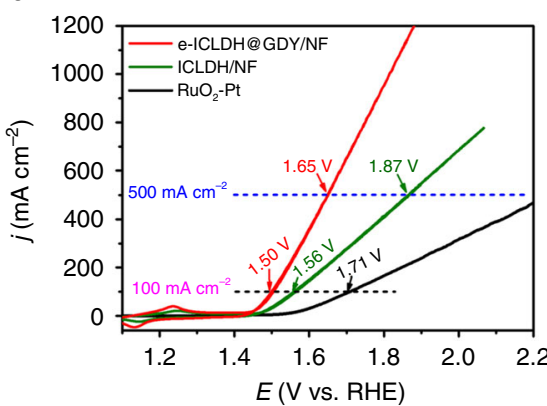

e

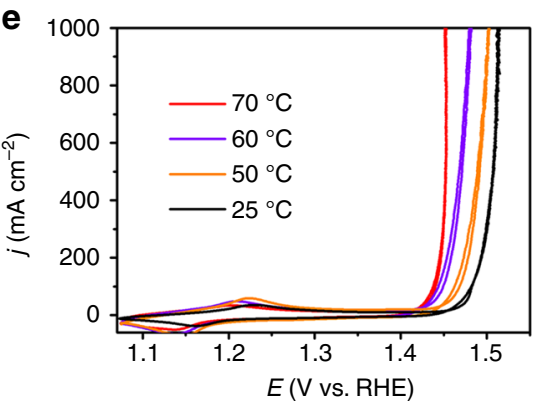

h

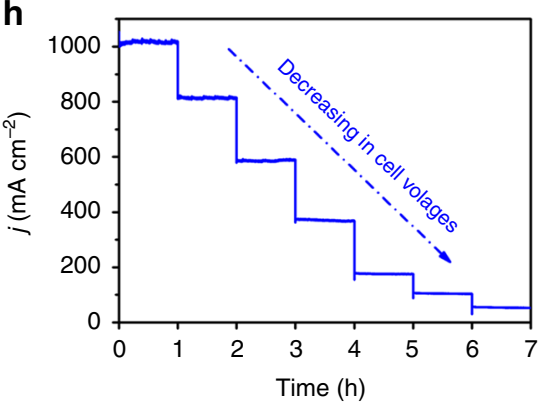

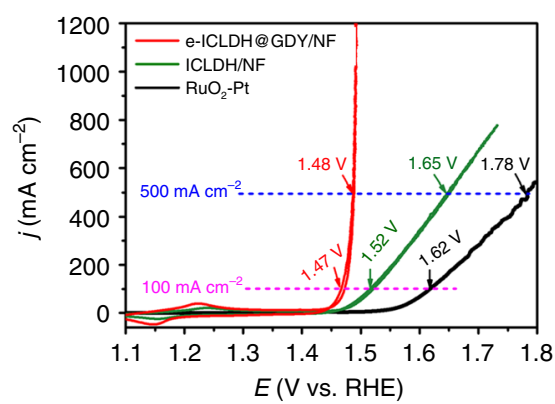

$\mathbf{f}$

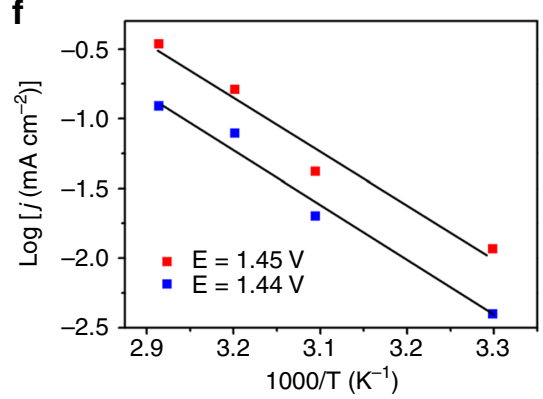

i

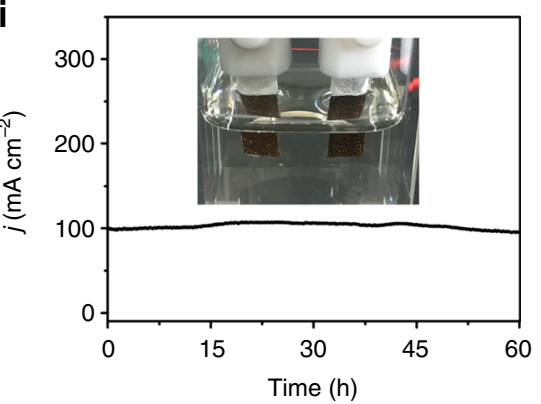

Fig. 7 OWS performance of the electrocatalyst. a Schematic representation of the OWS process. b, c OWS activities of e-ICLDH@GDY, a precious RuO ${ }_{2}$-Pt couple, and the pristine ICLDH/NF b without and $\mathbf{c}$ with iR-correction. $\mathbf{d}$ OWS activities of e-ICLDH@GDY and previously reported electrocatalysts. e CV curves for OWS processes performed at various temperatures. f Arrhenius plots for OWS processes performed on e-ICLDH@GDY/NF at various potentials. $\mathbf{g}$, $\mathbf{h}$ Current density-time curves measured with $\mathbf{g}$ increasing (red line) and $\mathbf{h}$ decreasing (blue line) cell voltages. $\mathbf{i}$ Current density of the eICLDH@GDY/NF electrode in an alkaline electrolyzer under a constant cell voltage of $1.56 \mathrm{~V}$, measured over $60 \mathrm{~h}$ (inset: the photograph for the twoelectrode system) 
$1.49 \mathrm{~V}$ to deliver 10,100 , and $1000 \mathrm{~mA} \mathrm{~cm}^{-2}$, respectively-that was much better than those of ICLDH/NF $(1.47$ and $1.52 \mathrm{~V}$ at 10 and $100 \mathrm{~mA} \mathrm{~cm}^{-2}$, respectively) and a $\mathrm{RuO}_{2}-\mathrm{Pt}$ couple (1.53 and $1.62 \mathrm{~V}$ at 10 and $100 \mathrm{~mA} \mathrm{~cm}^{-2}$, respectively), as well as those of other reported state-of-the-art electrodes, including $\mathrm{Co}_{1} \mathrm{Mn}_{1} \mathrm{CH}$ $\left(1.68 \mathrm{~V} \text { at } 10 \mathrm{~mA} \mathrm{~cm}^{-2}\right)^{10}, \mathrm{NiFeLDH} / \mathrm{NF}\left(1.70 \mathrm{~V} \text { at } 10 \mathrm{~mA} \mathrm{~cm}^{-2}\right)^{49}$, and P-NiFe $\left(1.51 \mathrm{~V} \text { at } 10 \mathrm{~mA} \mathrm{~cm}^{-2}\right)^{50}$ (Fig. 7d, Supplementary Table 5). The OWS reaction can be driven by a single-cell AA battery with a nominal voltage of $1.5 \mathrm{~V}$ (Supplementary Fig. 22, Supplementary Movie 1, 2).

Next, we examined the relationship between the temperature and the OWS activity of e-ICLDH@GDY/NF. As displayed in Fig. 7e, the cell voltage required to deliver a current density of $10 \mathrm{~mA} \mathrm{~cm}^{-2}$ decreased significantly from 1.43 to $1.41 \mathrm{~V}$ as the temperature increased from 25 to $70^{\circ} \mathrm{C}$. We extracted the kinetic barriers involved in the OWS process from the Arrhenius plots (Fig. 7f). The values of $E_{\mathrm{a}}$ for the OWS processes performed at cell voltages of 1.44 and $1.45 \mathrm{~V}$ (vs. RHE) were 77.22 and $74.34 \mathrm{~kJ} \mathrm{~mol}^{-1}$, respectively, revealing that the activation energy for the OWS process decreased upon increasing the cell voltage.

The water splitting capability of our electrolyzer was then examined at various cell voltages (Fig. $7 \mathrm{~g}, \mathrm{~h}$ ). Upon increasing the applied voltages, the current densities increased from approximately $50 \mathrm{~mA} \mathrm{~cm}^{-2}$ to approximately $1000 \mathrm{~mA} \mathrm{~cm}^{-2}$ and stabilized rapidly. The current densities decreased back from 1000 to $50 \mathrm{~mA} \mathrm{~cm}^{-2}$ upon decreasing the applied voltage, revealing the system's excellent stability. We attribute the small current fluctuations on the curves at large current densities to the rapid or vigorous evolution of gas bubbles from the electrode. We examined the long-term stability of the e-ICLDH@GDY/ $\mathrm{NF}$-based electrolyzer under a constant cell voltage of $1.56 \mathrm{~V}$. Figure $7 \mathrm{i}$ reveals that the current density of $100 \mathrm{~mA} \mathrm{~cm}^{-2}$ remained constant for more than $60 \mathrm{~h}$. The Faradaic efficiency of $\mathrm{O}_{2}$ evolution on e-ICLDH@GDY/NF was $97.40 \pm 1.30 \%$ (Supplementary Fig. 23). We observed vigorous evolution of gases from the electrode (inset in Fig. 7i), but no obvious fluctuations in the current density-time curve. Most impressively, e-LDH@GDY/NF required only 1.48 and $1.49 \mathrm{~V}$ to achieve stable current densities of 500 and $1000 \mathrm{~mA} \mathrm{~cm}^{-2}$, respectively, indicating that it is a very favorable electrocatalyst for rapid water splitting with possible practical applications.

We have prepared an active and stable bifunctional HER/ OER electrocatalyst, featuring GDY sandwiched between exfoliated ICLDH nanosheet arrays, through a GDY-induced intercalation-exfoliation method. This bifunctional electrocatalyst provided low OER overpotentials of 216,249 , and $278 \mathrm{mV}$ to reach current densities of 10,100 , and $1000 \mathrm{~mA} \mathrm{~cm}^{-2}$, respectively, and HER overpotentials of 43,215 , and $256 \mathrm{mV}$ to achieve current densities of 10,100 , and $1000 \mathrm{~mA} \mathrm{~cm}^{-2}$, respectively, in $1.0 \mathrm{M} \mathrm{KOH}$. It also displayed outstanding long-term stability, even when operated at a large current density of 1000 $\mathrm{mA} \mathrm{cm}^{-2}$ for both the OER $(>58 \mathrm{~h})$ and HER $(>67 \mathrm{~h})$. Impressively, when assembled into an alkaline electrolyzer, the electrocatalyst provided low voltages of $1.43,1.46$, and $1.49 \mathrm{~V}$ at current densities of 10,100 , and $1000 \mathrm{~mA} \mathrm{~cm}^{-2}$, respectively, for the OWS. Both experimental and theoretical data revealed that the incorporation of ICLDH with GDY improved the charge transport kinetics of the catalyst. Experimental results also confirmed that GDY played critical roles in enhancing the electrocatalytic activity and long-term stability of the electrocatalyst. Our synthetic strategy appears to be a simple and efficient method for in situ preparation of exfoliated LDH nanosheet arrays, without the need for additional binders.

\section{Methods}

The calculation details are given in the Supplementary Methods.

Synthesis of e-ICLDH@GDY/NF. ICLDH/NF was initially prepared via a hydrothermal method. Briefly, cobalt nitrate $(1 \mathrm{mmol})$, urea $(5 \mathrm{mmol})$, ferrous chloride $(0.25 \mathrm{mmol})$ and ammonium fluoride $(2 \mathrm{mmol})$ were added to a mixed solution of $\mathrm{H}_{2} \mathrm{O}(28 \mathrm{~mL})$ and ethanol $(5 \mathrm{~mL})$ under stirring for $30 \mathrm{~min}$. The mixture was then shifted to a $50 \mathrm{~mL}$ Teflon lined autoclave containing a piece of $\mathrm{Ni}$ foam $(2 \mathrm{~cm} \times 2 \mathrm{~cm})$ at $120^{\circ} \mathrm{C}$ for $8 \mathrm{~h}$. After $8 \mathrm{~h}$, the ICLDH/NF samples were taken out and washed with deionized water thoroughly and dried at $40^{\circ} \mathrm{C}$ in a vacuum oven. The obtained ICLDH/NF together with several pieces of $\mathrm{Cu}$ foils $(2.0 \times 2.0$ $\mathrm{cm}$ ) were added to a three-necked bottle containing acetone/pyridine/tetramethylethylenediamine $(100: 5: 1)$. HEB $(0.07 \mathrm{mmol})$ dissolved in pyridine $(50 \mathrm{~mL})$ was added very slowly into the above mixture. After a reaction at $50{ }^{\circ} \mathrm{C}$ for $25 \mathrm{~h}$ under Ar atmosphere, the e-ICLDH@GDY/NF was obtained. All products was washed with $2 \mathrm{M} \mathrm{HCl}, 2 \mathrm{M} \mathrm{NaOH}$, acetone, DMF and deionized water subsequently before use.

Characterization. The X-ray photoelectron spectroscopy (XPS, Thermo Scientific ESCALab 250Xi), FTIR spectroscopy (Thermo Fisher Nicolet6700), and Raman spectroscopy (Renishaw-2000) were used to get the structural information of samples. Field-emission SEM (Hitachi S-4800) and TEM, high-resolution TEM (JEM-2100F) were used to characterize the morphologies of the samples.

Electrochemical measurements. Electrochemical measurements were performed on a typical three-electrode system (CHI 760E, CH Instruments, Inc. Shanghai) using a saturated calomel electrode (SCE) as reference electrode, graphite rod as counter electrode, and the as-synthesized electrocatalyst as the working electrode. OER CV curves and HER polarization curves were recorded at $1 \mathrm{mV} \mathrm{s}^{-1}$ in $\mathrm{O}_{2^{-}}$ saturated and $\mathrm{H}_{2}$-saturated $1.0 \mathrm{M} \mathrm{KOH}$, respectively. Continuous cycling tests were carried out at $100 \mathrm{mV} \mathrm{s}^{-1}$. EIS data were collected at open-circuit potentials (frequency range: $100 \mathrm{KHz}$ to $0.1 \mathrm{~Hz}$ ) and fitted by an equivalent circuit $[\mathrm{R}(\mathrm{QR})(\mathrm{QR})]$ All potentials reported have been converted to the reversible hydrogen electrode $\left(\mathrm{RHE}, E_{\mathrm{RHE}}=E_{\mathrm{SCE}}+E_{(\mathrm{SCE})}^{0}+0.059 \mathrm{pH}\right)$.

\section{Data availability}

The data that support the plots within this paper and other finding of this study are available from the corresponding author upon reasonable request.

Received: 9 July 2018 Accepted: 20 November 2018

Published online: 14 December 2018

\section{References}

1. Walter, M. G. et al. Solar water splitting cells. Chem. Rev. 110, 6446-6473 (2010).

2. Ran, J., Zhang, J., Yu, J., Jaroniec, M. \& Qiao, S. Z. Earth-abundant cocatalysts for semiconductor-based photocatalytic water splitting. Chem. Soc. Rev. 43, 7787-7812 (2014)

3. Pham, T. A., Ping, Y. \& Galli, G. Modelling heterogeneous interfaces for solar water splitting. Nat. Mater. 16, 401-408 (2017).

4. $\mathrm{Hu}, \mathrm{C}$. et al. In situ electrochemical production of ultrathin nickel nanosheets for hydrogen evolution electrocatalysis. Chem 3, 122-133 (2017).

5. Gong, M. et al. An advanced Ni-Fe layered double hydroxide electrocatalyst for water oxidation. J. Am. Chem. Soc. 135, 8452-8455 (2013).

6. Hui, L. et al. Multifunctional single-crystallized carbonate hydroxides as highly efficient electrocatalyst for full water splitting. Adv. Energy Mater. 8, 1800175 (2018).

7. Jia, X. et al. $\mathrm{Ni}_{3} \mathrm{FeN}$ nanoparticles derived from ultrathin NiFe-layered double hydroxide nanosheets: an efficient overall water splitting electrocatalyst. Adv. Energy Mater. 6, 1502585 (2016).

8. Ma, Q. et al. Identifying the electrocatalytic sites of nickel disulfide in alkaline hydrogen evolution reaction. Nano Energy 41, 148-153 (2017).

9. Song, F. \& Hu, X. Exfoliation of layered double hydroxides for enhanced oxygen evolution catalysis. Nat. Commun. 5, 4477 (2014).

10. Tang, T. et al. Electronic and morphological dual modulation of cobalt carbonate hydroxides by Mn doping toward highly efficient and stable bifunctional electrocatalysts for overall water splitting. J. Am. Chem. Soc. 139, 8320-8328 (2017).

11. Ping, J. et al. Self-assembly of single-layer CoAl-layered double hydroxide nanosheets on 3D graphene network used as highly efficient electrocatalyst for oxygen evolution reaction. Adv. Mater. 28, 7640-7645 (2016).

12. Li, B., Xiao, J., Fu, Q. \& Bao, X. Confined catalysis under two-dimensional materials. Proc. Natl Acad. Sci. USA 114, 5930-5934 (2017). 
13. Long, M., Tang, L., Wang, D., Li, Y. \& Shuai, Z. Electronic structure and carrier mobility in graphdiyne sheet and nanoribbons: theoretical predictions. ACS Nano 5, 2593-2600 (2011).

14. Malko, D., Neiss, C., Viñes, F. \& Görling, A. Competition for graphene: graphynes with direction-dependent Dirac cones. Phys. Rev. Lett. 108, 086804-086807 (2012).

15. Haley, M. M. Synthesis and properties of annulenic subunits of graphyne and graphdiyne nanoarchitectures. Pure Appl. Chem. 80, 519-532 (2008).

16. Li, G. et al. Architecture of graphdiyne nanoscale films. Chem. Commun. 46, 3256-3258 (2010).

17. Li, Y. J., Xu, L., Liu, H. B. \& Li, Y. L. Graphdiyne and graphyne: from theoretical predictions to practical construction. Chem. Soc. Rev. 43, 2572-2586 (2014).

18. Li, Y.-j \& Li, Y.-1 Two dimensional polymers: progress of full carbon graphyne. Acta Polym. Sin. 2, 147-165 (2015).

19. Chen, Y., Huibiao, L. \& Li, Y. Progress and prospect of two dimensional carbon graphdiyne. Chin. Sci. Bull. 61, 2901-2912 (2016).

20. Huang, C.-S. \& Li, Y.-L. Structure of 2D graphdiyne and its application in energy fields. Acta Phys. -Chim. Sin. 32, 1314-1329 (2016).

21. Jia, Z. et al. Synthesis and properties of $2 \mathrm{D}$ carbon: graphdiyne. Acc. Chem Res. 50, 2470-2478 (2017).

22. Li, Y. Design and self-assembly of advanced functional molecular materials: from low dimension to multi-dimension. Sci. Sin. Chim. 47, 1045-1056 (2017).

23. Xue, Y. et al. Self-catalyzed growth of Cu@graphdiyne core-shell nanowires array for high efficient hydrogen evolution cathode. Nano Energy 30, 858-866 (2016).

24. Li, J. et al. Graphdiyne: a metal-free material as hole transfer layer to fabricate quantum dot-sensitized photocathodes for hydrogen production. J. Am. Chem. Soc. 138, 3954-3957 (2016).

25. Qian, X. et al. Self-catalyzed growth of large-area nanofilms of twodimensional carbon. Sci. Rep. 5, 7756 (2015).

26. Xue, Y., Li, Y., Zhang, J., Liu, Z. \& Zhao, Y. 2D Graphdiyne materials: challenges and opportunities in energy field. Sci. China Chem. 61, 765-786 (2018).

27. Huang, C. et al. Progress in research into $2 \mathrm{D}$ graphdiyne-based materials. Chem. Rev. 118, 7744-7803 (2018).

28. $\mathrm{Yu}, \mathrm{H}$. et al. Controlled growth of $\mathrm{MoS}_{2}$ nanosheets on $2 \mathrm{D} \mathrm{N}$-doped graphdiyne nanolayers for highly associated effects on water reduction. $A d v$. Func. Mater. 28, 1707564 (2018).

29. Yu, H. et al. Efficient hydrogen production on a 3D flexible heterojunction material. Adv. Mater. 30, 1707082 (2018).

30. Zhou, J. et al. Synthesis of graphdiyne nanowalls using acetylenic coupling reaction. J. Am. Chem. Soc. 137, 7596-7599 (2015).

31. Gao, X. et al. Direct synthesis of graphdiyne nanowalls on arbitrary substrates and its application for photoelectrochemical water splitting cell. Adv. Mater. 29, 1605308 (2017).

32. Kloprogge, J., Wharton, D., Hickey, L. \& Frost, R. Infrared and Raman study of interlayer anions $\mathrm{CO}_{3}{ }^{2-}, \mathrm{NO}_{3}{ }^{-}, \mathrm{SO}_{4}{ }^{2-}$ and $\mathrm{ClO}_{4}{ }^{-}$in $\mathrm{Mg} / \mathrm{Al}$-hydrotalcite. Am. Mineral. 87, 623-629 (2002).

33. Liu, P. F., Yang, S., Zhang, B. \& Yang, H. G. Defect-rich ultrathin cobalt-iron layered double hydroxide for electrochemical overall water splitting. ACS Appl. Mater. Interfaces 8, 34474-34481 (2016).

34. Yeo, B. S. \& Bell, A. T. Enhanced activity of gold-supported cobalt oxide for the electrochemical evolution of oxygen. J. Am. Chem. Soc. 133, 5587-5593 (2011).

35. Kong, X., Huang, Y. \& Liu, Q. Two-dimensional boron-doped graphyne nanosheet: A new metal-free catalyst for oxygen evolution reaction. Carbon 123, 558-564 (2017).

36. Zhao, S. et al. Gold nanoclusters promote electrocatalytic water oxidation at the nanocluster/CoSe ${ }_{2}$ interface. J. Am. Chem. Soc. 139, 1077-1080 (2017).

37. Xue, Y. et al. Anchoring zero valence single atoms of nickel and iron on graphdiyne for hydrogen evolution. Nat. Commun. 9, 1460 (2018).

38. Zhang, J. et al. Efficient hydrogen production on MoNi4 electrocatalysts with fast water dissociation kinetics. Nat. Commun. 8, 15437 (2017).

39. $\mathrm{Yu}, \mathrm{L}$. et al. Ternary $\mathrm{Ni}_{2(1-\mathrm{x})} \mathrm{Mo}_{2 \mathrm{x}} \mathrm{P}$ nanowire arrays toward efficient and stable hydrogen evolution electrocatalysis under large-current-density. Nano Energy 53, 492-500 (2018).

40. Zhou, H. et al. Water splitting by electrolysis at high current density under 1.6 volt. Energy Environ. Sci. 11, 2858-2864 (2018).

41. Jin, $\mathrm{H}$. et al. In situ cobalt-cobalt oxide/ $\mathrm{N}$-doped carbon hybrids as superior bifunctional electrocatalysts for hydrogen and oxygen evolution. J. Am. Chem. Soc. 137, 2688-2694 (2015).
42. Chen, Z., Kronawitter, C. X. \& Koel, B. E. Facet-dependent activity and stability of $\mathrm{Co}_{3} \mathrm{O}_{4}$ nanocrystals towards the oxygen evolution reaction. Phys. Chem. Chem. Phys. 17, 29387-29393 (2015).

43. Zhu, C. et al. Nickel cobalt oxide hollow nanosponges as advanced electrocatalysts for the oxygen evolution reaction. Chem. Commun. 51, 7851-7854 (2015).

44. Dong, X. et al. 3D graphene-cobalt oxide electrode for high-performance supercapacitor and enzymeless glucose detection. ACS Nano 6, 3206-3213 (2012).

45. Zhang, K., Hu, Z., Liu, X., Tao, Z. \& Chen, J. FeSe ${ }_{2}$ microspheres as a highperformance anode material for Na-ion batteries. Adv. Mater. 27, 3305-3309 (2015).

46. Meher, S. K. \& Rao, G. R. Ultralayered $\mathrm{Co}_{3} \mathrm{O}_{4}$ for high-performance supercapacitor applications. J. Phys. Chem. C 115, 15646-15654 (2011).

47. Pendashteh, A., Palma, J., Anderson, M. \& Marcilla, R. Nanostructured porous wires of iron cobaltite: novel positive electrode for highperformance hybrid energy storage devices. J. Mater. Chem. A 3, 16849-16859 (2015).

48. Zhang, B. et al. Homogeneously dispersed, multimetal oxygen-evolving catalysts. Science 352, 333-337 (2016).

49. Luo, J. et al. Water photolysis at $12.3 \%$ efficiency via perovskite photovoltaics and earth-abundant catalysts. Science 345, 1593-1596 (2014).

50. Zhang, F.-S. et al. Extraction of nickel from NiFe-LDH into $\mathrm{Ni}_{2} \mathrm{P} @ \mathrm{NiFe}$ hydroxide as a bifunctional electrocatalyst for efficient overall water splitting. Chem. Sci. 9, 1375-1384 (2018).

\section{Acknowledgements}

This work was supported by the National Nature Science Foundation of China (21790050 and 21790051), the National Key Research and Development Project of China (2016YFA0200104), and the Key Program of the Chinese Academy of Sciences (QYZDYSSW-SLH015).

\section{Author contributions}

Yuliang Li conceived and designed the research and analysis, reviewed and edited this manuscript. L.H. carried out the catalyst synthesis, electrochemical experiments and wrote corresponding discussions. Y.X. helped to collect, analyze and organize the data, and write the draft. B.H. gave important help in theoretical calculations. H.Y., C.Z., D.Z., D.J., Y.Z., Yongjun Li and H.L. gave helpful advice.

\section{Additional information}

Supplementary Information accompanies this paper at https://doi.org/10.1038/s41467018-07790-x.

Competing interests: The authors declare no competing interests.

Reprints and permission information is available online at http://npg.nature.com/ reprintsandpermissions/

Publisher's note: Springer Nature remains neutral with regard to jurisdictional claims in published maps and institutional affiliations.

Open Access This article is licensed under a Creative Commons Attribution 4.0 International License, which permits use, sharing, adaptation, distribution and reproduction in any medium or format, as long as you give appropriate credit to the original author(s) and the source, provide a link to the Creative Commons license, and indicate if changes were made. The images or other third party material in this article are included in the article's Creative Commons license, unless indicated otherwise in a credit line to the material. If material is not included in the article's Creative Commons license and your intended use is not permitted by statutory regulation or exceeds the permitted use, you will need to obtain permission directly from the copyright holder. To view a copy of this license, visit http://creativecommons.org/ licenses/by/4.0/.

(c) The Author(s) 2018 